\title{
Armando Bauleo, in memoriam
}

Armando Bauleo era psiquiatra, psicoanalista, psicólogo social y autor de numerosos libros prestigiosos sobre Grupos, entre ellos, los más conocidos: Ideología, grupo y familia (1970); Contrainstitución y grupos (1977); La Concepción operativa de grupo (1990); Notas de psicología y Psiquiatría social (1988); Psicoanálisis operativo (2005), Psicoanálisis y grupalidad (2007). Asimismo escribió numerosos artículos en libros y revistas profesionales. En Argentina -su tierra natal-, Armando trabajó y escribió con Pichón Rivière (uno de sus escritos conjuntos más conocidos, La noción de tarea en Psiquiatría está incluido en el libro de Pichón Rivière El Proceso grupal). Bauleo fue uno de sus discípulos más destacados y quien continuó su trabajo de creación e investigación en el Campo de lo Grupal. En 1976 viene exiliado de la dictadura argentina; allí era miembro de la Asociación Psicoanalítica Argentina, perteneciente a la IPA, a la que renunció junto a otros miembros titulares y adherentes y otros reconocidos profesionales. Fundaron el grupo Plataforma, cuyo objetivo era introducir el Psicoanálisis en la realidad social. En nuestro país trabajó y vivió varios años, formó a muchos profesionales que han adquirido responsabilidades importantes en el Campo de la Salud Mental, muchos de ellos socios de la AEN, de cuya Revista fue colaborador desde el año 1987.

Recibo la noticia de la muerte de Armando Bauleo desconcertada, sin poder creer que a un hombre con tanta energía y vitalidad, como lo atestiguan sus continuos viajes de un país y de un continente a otro para transmitir su experiencia y saber profesional, le pueda llegar este momento. Cuando tomo conciencia de su muerte me aflige un profundo dolor por los momentos vividos con él, tanto profesionales como personales, por ello quisiera recordar su figura a través de las experiencias compartidas desde inicios de 1978. Previamente es preciso situar su llegada a España para comprender el lugar y la importancia de Bauleo en nuestro país. Había llegado expatriado de la dictadura de su país a una España que, a su vez, salía de 40 años de dictadura franquista. España en ese momento trataba de reconstituirse, tenía una asistencia psiquiátrica básicamente asilar, no había ningún desarrollo de los Centros de Salud Mental, sólo existían ambulatorios donde la atención era masificada y donde, los neuropsiquiatras, en un horario laboral de dos horas, atendían psicofarmacológicamente a los pacientes. Una de las honrosas excepciones era el Instituto Peña Retama, comunidad terapéutica que trabajaba con grupos psicoterapéuticos, su fundador Molina Núñez se había formado también con el Dr. Pichón Rivière.

En este contexto social de vacío cultural, asistencial, de formación e investigación psicológica y psiquiátrica de los profesionales, pudimos compartir con él numerosos intereses, los primeros fueron los referentes al movimiento antipsi- 
quiátrico que tenía tanta fuerza en el sur de Europa y algunos países de Latinoamérica. En España el referente era el hospital de día de Psiquiatría del actual hospital Gregorio Marañón, antes denominado Francisco Franco, dirigido por González Duro, y cuya experiencia viví desde su fundación en 1974 hasta 1977. Bauleo organizó el primer viaje profesional del psiquiatra italiano Basaglia a Madrid, ambos estaban unidos por una excelente amistad y el interés por la desmanicomialización del loco. Basaglia era el representante del movimiento antipsiquiátrico en Italia y consiguió importantes reformas legales y asistenciales en la atención de los enfermos mentales de su país, fruto de su trabajo en el setenta y ocho es la Ley 180 que suprimía la utilización del hospital psiquiátrico.

Antes de este encuentro, «mi pasión por los grupos» es lo que me había llevado a conocer a Armando y lo que me llevó a iniciar mi formación con él en grupo operativo, que se prolongó durante bastantes años. Los Grupos Operativos, en palabras del autor, habían nacido a inicios de los años cincuenta, en un momento de crítica a la dicotomía entre lo endógeno y lo exógeno en el origen de la enfermedad mental, en la hipótesis de la multicausalidad o la complejidad de lo causal, lo que conllevaba una diferente concepción del proceso del enfermarse y del de la curación. Los conceptos psicoanalíticos habían revolucionado el campo de la psiquiatría, la búsqueda del sentido de los síntomas y la interpretación eran centrales en el proceso terapéutico. En este planteamiento teórico, la evolución del cuadro no era lineal, sino que iba a depender de diversos factores, entre ellos, la subjetividad y formación del terapeuta. Para Bauleo, la elección de lo Grupal se enmarcaba dentro de una concepción diferente de la Psiquiatría y de la clínica, el grupo surgía como instrumento privilegiado en el diagnóstico, prevención y tratamiento de la Psiquiatría y de la Salud Mental. En aquellos años para los españoles, los grupos operativos que realizamos con él eran un aprendizaje totalmente novedoso, hacíamos pequeños grupos que se estructuraban alrededor de una tarea explícita: Teorías Grupales y Concepción Operativa de Grupo, un aprendizaje en el que la articulación entre el pensamiento y lo afectivo era central. Simultaneábamos esta formación con el estudio de la Teoría Psicoanalítica a cargo de su mujer, Marta de Brasi y Armando Ingala, director del CEPYP, y, a su vez, hacíamos la experiencia de nuestro propio análisis individual.

Conocerle, y elegir mi formación profesional con Bauleo fue muy importante para mi y para muchos profesionales psiquiatras, psicólogos, enfermeras, asistentes sociales que trabajamos actualmente en los Equipos de los Centros de Salud Mental en España, ya que Armando fue uno de los primeros maestros en la enseñanza de las Teorías Grupales e Institucionales, desde un esquema referencial psicoanalítico, que nos permitió aprehender de otra manera la enfermedad y la salud mental. Nos enseñó el funcionamiento de lo colectivo, de la familia, de la 
relación entre ésta y la salud mental de un sujeto, aprendimos que el paciente era «emergente» del grupo familiar. Para él la psicopatología se manifestaba en un sujeto, pero estaba siempre en relación a su situación grupal, familiar y laboral; por tanto, el instrumento grupal era fundamental en su tratamiento. Este aprendizaje grupal nos permitió conocer y trabajar con profesionales interesados en los cambios institucionales y en la reforma de la Asistencia Psiquiátrica y de la Salud Mental en España, algunos de ellos hemos sido miembros de las juntas directivas de la AEN, representantes en la Comisión Nacional de la Especialidad de Psiquiatría y Psicología, responsables de Salud Mental en la Agencia Laín Entralgo, en las Juntas y Revistas del Colegio Oficial de Psicólogos, y en los Centros de Salud Mental.

En el setenta y nueve tuve el honor de organizar con él las Primeras Jornadas en Madrid de la Asociación Iberoamericana de Grupo, cuyo tema fue $L a$ Concepción Operativa de Grupo, y posteriormente, la suerte de coordinar con él grupos de formación que se habían organizado en algunas ciudades de España e Italia (Bolonia y Venecia). En esta ciudad Armando había fijado su residencia después de vivir unos años en Madrid y en Milán. Cuando se fue a trabajar a Italia, me encargó ser su enlace con el grupo de Madrid. Nuestro grupo estudiaba e investigaba, y él venía a seguir formándonos y a participar como co-coordinador y supervisor del trabajo que realizábamos como coordinadores de los grupos de formación y de los grupos terapéuticos que organizamos en torno al Instituto de Psicología Social y Grupo Operativo y en el Centro de Psicoterapia Grupal. Supervisó el trabajo clínico, grupal e institucional de numerosos profesionales de la salud mental en diversas ciudades españolas, también de algunos equipos de Centros de Salud Mental, en Madrid (Getafe), Andalucía y Zaragoza. Para él era fundamental introducir metodologías grupales en la organización y el quehacer de la práctica clínica en las Instituciones de Salud Mental, objetivo prioritario que hoy en día perdura en algunos equipos, ya que aún trabajan con este modelo grupal. También participó en los programas de docencia de los residentes de psiquiatría. Como tutora de MIR en el Centro de Salud Mental de Parla, área X de la CAM, le invité en dos ocasiones a Leganés, lugar donde se centralizaba toda la docencia de la Zona Sur de Madrid

Para Armando lo más importante en esos años fue la creación y fundación del Centro Internacional de Investigación en Psicología Social y Grupal, cuya primera reunión se celebró en Milán en 1981 y donde acepté estar junto a él en el primer Secretariado del Centro. El CIR reunía a profesionales de diversas ciudades europeas y latinoamericanas y en sus diversos boletines los profesionales pudimos escribir sobre nuestras experiencias realizadas con grupos. Yo lo hice, entre otros, con médicos de atención primaria, cuya tarea era trabajar sobre «la relación médi- 
co-paciente», tema de mi posterior Tesis Doctoral. Armando nos enseñaba una nueva forma de trabajo en la clínica psiquiátrica y en salud mental en la que esta relación era nuclear y fundamental para la evolución de la enfermedad. En estos años señalar también el importante papel de Armando en el proyecto del Centro de Medicina Comunitaria, ubicado en el hospital Doce de Octubre, que también fue una experiencia muy significativa para todos nosotros, en ella aprendimos la importancia de lo comunitario, de lo interdisciplinario, del trabajo en equipo, así como la necesidad y dificultad de los cambios institucionales y grupales.

En el año noventa, Bauleo coordinó una de las ponencias del XVIII Congreso de la AEN que se celebró en Madrid y que fue publicada con el título, $L a$ Concepción Operativa de Grupo, en la que participé hablando de «Clínica Grupal», espacio de reflexión sobre la salud y la enfermedad mental. Para Bauleo «la Clínica Grupal nacía de la convergencia de pensamientos analíticos freudianos, de grupo operativo y de psicodrama». La pasión de Bauleo en pensar la grupalidad de la enfermedad, del contexto terapéutico, de la organización de la institución, nos permitió vivenciar que había otra manera de organización de los servicios, que se podía trabajar en equipos interdisciplinarios y que el quehacer clínico podía priorizar los factores psicológicos y sociales de los pacientes sin negar la importancia de lo psicofarmacológico. Por desgracia, este modelo de trabajo en los Centros de Salud Mental actualmente es minoritario, ya que la eficacia de los nuevos psicofármacos, con pocos efectos secundarios y fácil prescripción, ha influido para que se receten masivamente. Estos resultados también han determinado que se priorice el origen biológico de la enfermedad mental en detrimento del análisis de los factores psicológicos y sociales y del buen quehacer psicoterapéutico individual y grupal, medicalizando, a veces, problemáticas de la vida cotidiana.

Armando también nos animó a publicar nuestras experiencias, de este modo escribí junto a Susana Amilibia, Mujer, deseo y embarazo, que él mismo presentó en el Instituto de la Mujer, en el año 1985. El libro estaba prologado por Marie Langer, a quien le unía una gran amistad, conocida psiquiatra, psicoanalista, miembro fundadora de la Asociación Psicoanalítica Argentina y posteriormente miembro de Plataforma y autora del excelente libro Maternidad y sexo. Cuando muere Marie Langer, Armando me invitó a participar en el acto de homenaje por la muerte de esta mujer luchadora, que había trabajado en numerosos campos, entre ellos, el de la mujer. El acto se celebró en Madrid, y participaron además del propio Bauleo, Grinberg, un representante de la AEN y del colegio Oficial de Psicólogos y un representante del Partido de los Trabajadores.

En 1997, otra publicación compartida con Armando fue Grupos Terapéuticos y Asistencia Pública, editado por la AEN, que coordiné junto a Emilio Gamo y en 
el que participaron, entre otros, Nicolás Caparros, Nicolás Espiro, Robert Lévy, Alejando Ávila y Alejandro Scherzer, todos ellos autores reconocidos en el campo de lo grupal. En estos últimos años Bauleo desarrolló su trabajo en Italia como Director científico en el Instituto de Psicología Social Analítica en Venecia y, en España, en la Asociación de Psicoterapia Operativa Psicoanalítica, experiencias de las que hablaran otros compañeros italianos y españoles en sus revistas profesionales de referencia. La última participación significativa en Madrid se produce hace dos años, en 2006, donde Bauleo pronuncia la conferencia inaugural del Congreso Internacional sobre La actualidad del grupo operativo, al que lamentablemente no pude asistir, organizado por la Asociación para el Estudio de Temas Grupales, Psicosociales e Institucionales y el Espacio de Estudio sobre la Grupalidad, asociaciones que trabajan en España sobre el pensamiento grupal y la psicología social siguiendo los postulados de Pichón Rivière.

Armando, que sirva este in memoriam para rememorar tu labor como intelectual, autor, profesor, clínico, investigador, supervisor y formador de alumnos y compañeros repartidos en medio mundo, así como para recordarte con todo mi cariño y reconocimiento. Tu contribución al desarrollo de la Clínica Grupal y del trabajo en Equipo e Institucional queda en el quehacer de los profesionales que fuimos alumnos tuyos. Estas palabras han servido para despedirme, para separarme de ti Armando, aunque tu recuerdo siempre estará vivo dentro de mí. En recuerdo por tantos buenos momentos pasados juntos, en los que nos contagiabas tu alegría de vivir y tu placer por el trabajo, cuando fundamos el CIR, en los Congresos, en las supervisiones, y por tantas horas grupales inolvidables en los grupos de formación que coordinabas, donde nos asombrábamos por la maestría en tu quehacer y en la lectura de lo latente grupal.

Gracias por tus enseñanzas, nos queda tu afecto y tu obra. Tu recuerdo queda vivo en mi memoria y en el trabajo de tantos de nosotros. 